\title{
ACID BASE STATUS AND CEREBRAL OXYGENATION IN NEONATES IMMEDIATELY AFTER BIRTH
}

Gerhard Pichler ${ }^{1,2}$, Berndt Urlesberger ${ }^{1,2}$, Christian Mattersberger ${ }^{1,2}$, Nariae Baik-Schneditz ${ }^{1,2}$, Bernhard Schwaberger $^{1,2}$, Lukas Mileder ${ }^{1,2}$, Georg Schmölzer ${ }^{3,4}$

\author{
1 Research Unit for Neonatal Micro- and Macrocirculation, Department of Pediatrics and Adolescent Medicine, Medical University of Graz, Austria. \\ $\underline{2 \text { Division of Neonatology, Department of Pediatrics and Adolescent Medicine, Medical University of Graz, Graz, Austria. }}$ \\ 3 Centre for the Studies of Asphyxia and Resuscitation, Royal Alexandra Hospital, Edmonton, Canada \\ 4 Department of Pediatrics, University of Alberta, Edmonton, Canada
}

Background and Aim: Cerebral tissue oxygenation in preterm and term neonates is of growing interest. There are no data available concerning influences of acid base status on cerebral tissue oxygenation during immediate transition after birth.

Aim of the present study was therefore to investigate possible associations of acid base status and cerebral tissue oxygenation in preterm and term neonates 15 minutes after birth.

\section{Methods:}

Analyses of secondary outcome parameters of prospective observational studies.

Preterm and term neonates were included, in whom

1. cerebral regional tissue oxygenation ( $\mathrm{crSO}$ ) was measured during immediate transition after birth and

2. blood samples for blood gas analyses were taken between 15 to 20 minutes after birth

- arterial oxygen saturation (SpO2) and heart rate were measured by pulse oximeter on the right wrist

- cerebral fractional-tissue-oxygen-extraction (cFTOE) was calculated out of SpO2 and crSO2

- correlation analyses to investigate potential associations between crSO2/CFTOE at minute 15 after birth and acid base status (ph-value, base excess (BE), standard bicarbonate ( $\mathrm{HCO})$ ) were performed.

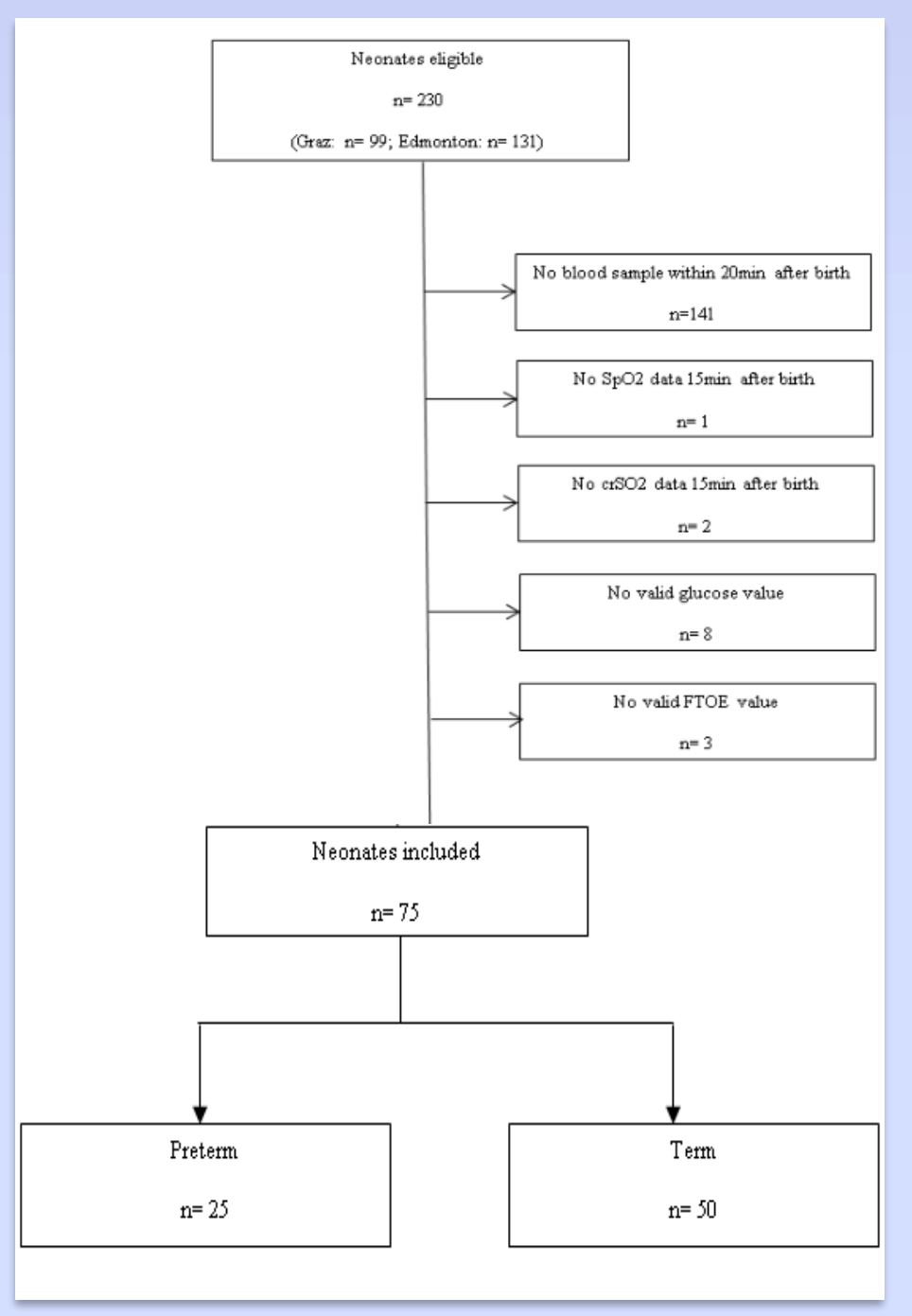

\begin{tabular}{|c|c|c|c|}
\hline & Preterm & Term & $\mathrm{p}$-Value \\
\hline Gestational age (weeks) & $\frac{n=25}{328 \pm 33}$ & & \\
\hline Birthweight (gram) & $1757 \pm 545$ & $3149 \pm 524$ & $<0.001$ \\
\hline APGAR 1 & $7.7 \pm 1.9$ & $8.7 \pm 0.9$ & $<0.001$ \\
\hline APGAR 5 & $8.8 \pm 1.5$ & $9.7 \pm 0.6$ & $<0.001$ \\
\hline APGAR 10 & $9.3 \pm 0.9$ & $9.9 \pm 0.2$ & $<0.001$ \\
\hline Umbilical artery $\mathrm{pH}$ value & $7.3 \pm 0.05$ & $7,3 \pm 0.04$ & 0.551 \\
\hline Mean arterial blood pressure $(\mathrm{mmHg}) *$ & $44 \pm 9$ & $46 \pm 8$ & 0.218 \\
\hline Central temperature $\left({ }^{\circ} \mathrm{C}\right) *$ & $36.8 \pm 0.3$ & $37 \pm 0.3$ & 0.058 \\
\hline Supplemental oxygen, $\mathrm{n}(\%)$ & $11(44)$ & 1 (2) & $<0.001$ \\
\hline Non-invasive respiratory support, $\mathrm{n}(\%)$ & $12(48)$ & 6 (12) & $<0.001$ \\
\hline Intubation, $\mathbf{n}(\%)$ & $1(4)$ & $0(0)$ & \\
\hline Arterial oxygen saturation (\%) * & $93.9 \pm 5.1$ & $96 \pm 3.2$ & 0.058 \\
\hline Heart rate $(\mathrm{bpm}) *$ & $159 \pm 15$ & $157.1 \pm 19$ & 0.646 \\
\hline Cerebral regional oxygen saturation $(\%) *$ & $80.2 \pm 12.1$ & $83 \pm 7.7$ & 0.382 \\
\hline Fractional tissue oxygen extraction * & $0.15 \pm 0.10$ & $0.14 \pm 0.08$ & 0.521 \\
\hline Blood glucose concentration (mmollit) & $2.7 \pm 0.9$ & $2.9 \pm 0.9$ & 0.079 \\
\hline Blood glucose concentration (mg/dlit) & $57.5 \pm 14.1$ & $53 \pm 9.2$ & 0.079 \\
\hline Blood sample p.p. time (min) & $17,0 \pm 2,0$ & $15.8 \pm 1.3$ & 0.008 \\
\hline Lactate concentration (mmolil $)$ & $2.7 \pm 0.8$ & $2.8 \pm 0.9$ & 0.760 \\
\hline Base excess (mmoll $\mathrm{L}$ ) & $-2.07 \pm 2.5$ & $-1.4 \pm 2$ & 0.232 \\
\hline Standardized bicarbonate (mmolli) & $21.5 \pm 2.6$ & $21.4 \pm 1.7$ & 0.699 \\
\hline pH Value & $7.29 \pm 0.1$ & $7.28 \pm 0.1$ & 0.722 \\
\hline
\end{tabular}

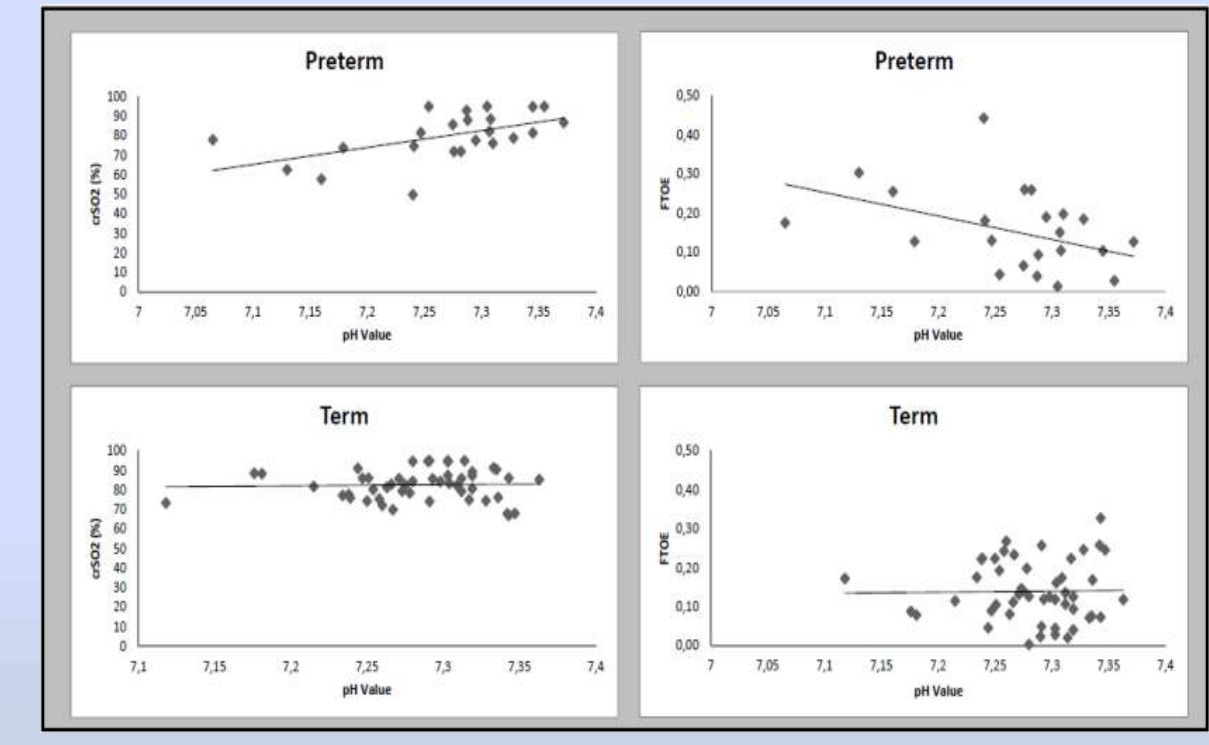

Preterm neonates:

(A) Regional cerebral oxygen saturation (crSO2, \%) and $p H$ value; $q=0,57, p=0.01$ Term neonates: Term neonates:
(C) Regional cerebral oxygen saturation (crSO2, \%) and $\mathrm{pH}$ value; $\mathrm{q}=0.03, \mathrm{p}=0.82$

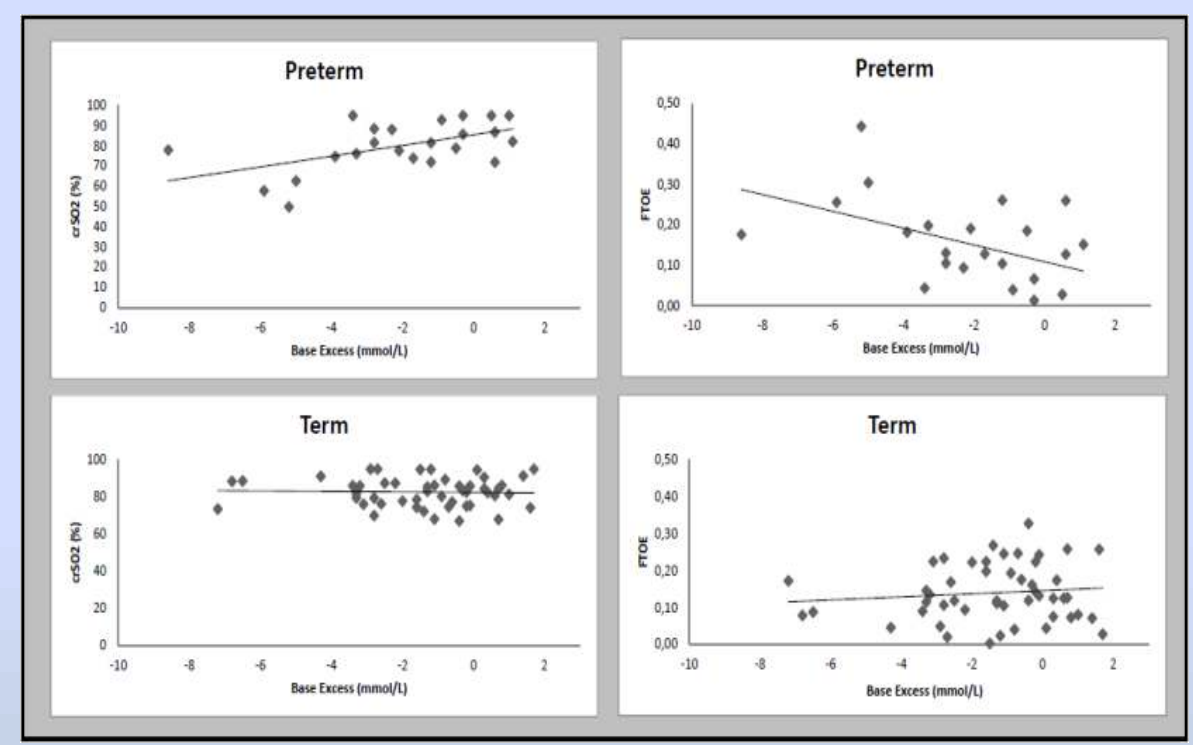

Preterm neonates:

(A) Regional cerebral oxygen saturation (crSO2, \%) and base excess ( $m$ mol/L); $\mathrm{q}=0,54, \mathrm{p}=0,01$
(B) Fractional tissue oxygen satuation (FTOE) and base excess (mmo/L); $\mathrm{q}=-0,49, \mathrm{p}=0,02$ Term neonates: (C) Regional cerebral oxygen saturation (crSO2, \%) and base excess (mmo/l/L); $\mathrm{q}=-0,03, \mathrm{p}=0.82$

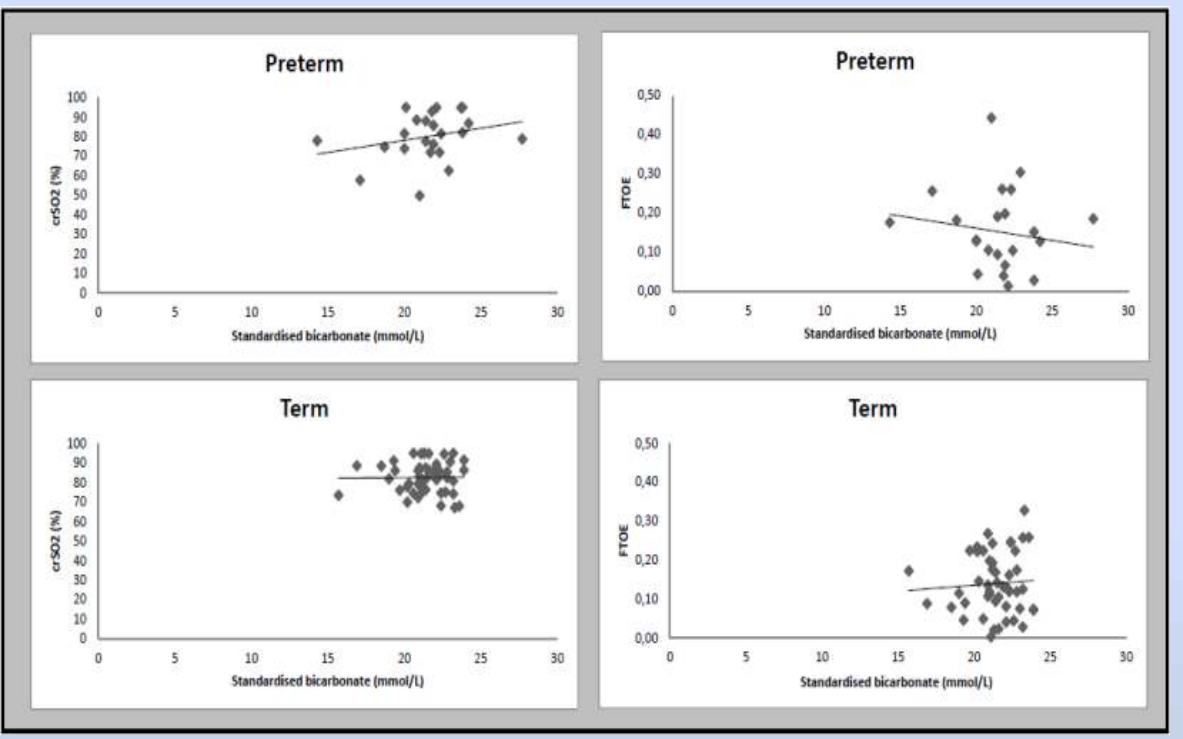

Preterm neonates:
(A) Regional cerebral oxygen saturation (crSO2, \%) and standardised bicarbonate ( $m$ mol/L); $q=0,27, p=0.21$
(B) Fractional tissue oxygen extraction (FTOE) and standardised bicarbonate (mmol/L) $q=0.15, p=0.48$ Term neonates:

Term neonates:
(C) Regional cerebral oxygen saturation (crSO2, \%) and standardised bicarbonate (mmol/L); $q=0.015, \mathrm{p}=0.92$
(D) Fractional tissue oxygen extraction (FTOE) and standardised bicarbonate (mmo/l/L); $q=0.03, \mathrm{p}=0.85$

\section{Conclusion:}

In preterm neonates immediately after birth there are associations of cerebral oxygenation and acid base status parameters, whereas in term neonates these associations cannot be observed. 